\title{
Inactivation of Basolateral Amygdala Specifically Eliminates Palatability-Related Information in Cortical Sensory Responses
}

\author{
Caitlin E. Piette, ${ }^{1,2}$ Madelyn A. Baez-Santiago, ${ }^{2}$ Emily E. Reid, ${ }^{3}$ Donald B. Katz, ${ }^{1,2,3 *}$ and Anan Moran ${ }^{2,3 *}$ \\ ${ }^{1}$ Program of Neuroscience, ${ }^{2}$ Volen Center for Complex Systems, and ${ }^{3}$ Department of Psychology, Brandeis University, Waltham, Massachusetts 02454
}

Evidence indirectly implicates the amygdala as the primary processor of emotional information used by cortex to drive appropriate behavioral responses to stimuli. Taste provides an ideal system with which to test this hypothesis directly, as neurons in both basolateral amygdala (BLA) and gustatory cortex (GC) - anatomically interconnected nodes of the gustatory system - code the emotional valence of taste stimuli (i.e., palatability), in firing rate responses that progress similarly through "epochs." The fact that palatability-related firing appears one epoch earlier in BLA than GC is broadly consistent with the hypothesis that such information may propagate from the former to the latter. Here, we provide evidence supporting this hypothesis, assaying taste responses in small GC single-neuron ensembles before, during, and after temporarily inactivating BLA in awake rats. BLA inactivation (BLAx) changed responses in $98 \%$ of taste-responsive GC neurons, altering the entirety of every taste response in many neurons. Most changes involved reductions in firing rate, but regardless of the direction of change, the effect of BLAx was epoch-specific: while firing rates were changed, the taste specificity of responses remained stable; information about taste palatability, however, which normally resides in the "Late" epoch, was reduced in magnitude across the entire GC sample and outright eliminated in most neurons. Only in the specific minority of neurons for which BLAx enhanced responses did palatability specificity survive undiminished. Our data therefore provide direct evidence that BLA is a necessary component of GC gustatory processing, and that cortical palatability processing in particular is, in part, a function of BLA activity.

\section{Introduction}

The brain consists of clusters of anatomically distinct but physically and functionally interconnected neurons. Countless studies have examined the perceptual functions of individual clusters (single brain regions), but far less work has investigated how separated clusters cooperate to yield complex behaviors. Cooperation between amygdala and cortex (Ottersen, 1982; Saper, 1982; Yamamoto et al., 1984) has been the subject of a fair amount of recent empirical inquiry, however; researchers have recently suggested that the processing of emotionally laden stimuli is an explicit amygdala-cortical function, with amygdala processing emotional information then used by cortex to drive appropriate behavioral responses to the stimuli (Schoenbaum et al., 1998; Bauer et al., 2007; Paz et al., 2009; Popa et al., 2010).

The taste system provides an ideal arena in which to explore this hypothesis. Gustatory cortex (GC) and basolateral amygdala (BLA) are monosynaptically (Saper, 1982; Shi and Cassell, 1998;

Received Feb. 12, 2012; revised May 29, 2012; accepted May 30, 2012.

Author contributions: C.E.P. and D.B.K. designed research; C.E.P., M.A.B.-S., and E.E.R. performed research; C.E.P. and A.M. analyzed data; C.E.P. and D.B.K. wrote the paper.

This work was supported by the National Institute on Deafness and Other Communication Disorders, Grants DC-006666 (D.B.K.) and DC-011235 (C.E.P.), and a grant from the Swartz Foundation for Computational Neuroscience (A.M.).

${ }^{*}$ D.B.K. and A.M. contributed equally to this work.

Correspondence should be addressed to Donald B. Katz, Department of Psychology, Brandeis University, 415 South Street, Waltham MA 02454.E-mail: dbkatz@brandeis.edu.

DOI:10.1523/JNEUROSCI.0669-12.2012

Copyright $\odot 2012$ the authors $\quad 0270-6474 / 12 / 329981-11 \$ 15.00 / 0$
Stone et al., 2011) and polysynaptically connected (Norgren and Leonard, 1973; Karimnamazi and Travers, 1998) structures that appear to cooperate for purposes of taste perception and learning (Escobar et al., 1998a,b; Ferreira et al., 2005; Grossman et al., 2008; Fontanini et al., 2009).

Examinations of taste response dynamics reveal details of what this cooperative amygdala-cortical taste coding might look like. GC taste responses progress through sequences of epochs (taste-specific spike rates with particular response latencies and durations), which code first the presence, then the identity, and finally the palatability of tastes (Erickson et al., 1994; Katz et al., 2001; Bahar et al., 2004; Jones et al., 2007) in the time preceding the onset of taste-specific behaviors (Travers et al., 1987; Fontanini and Katz, 2006). Response dynamics in BLA are a close match for those of GC, in that BLA neurons switch through epochs at similar times. Palatability-related information appears one epoch earlier in BLA, however (Fontanini et al., 2009); thus, the processing of the emotional properties of tastes could be argued to begin in BLA, and to "spread" to cortex one epoch later, in what appears to be a specific instantiation of the general hypothesis concerning amygdala-cortical cooperation.

The above evidence is purely phenomenological, however. More direct testing of this hypothesis requires an experiment combining multielectrode electrophysiology (Yamamoto, 1984; Katz et al., 2001) and pharmacological perturbation (Wang et al., 2006; Fortis-Santiago et al., 2010; Parkes and Westbrook, 2010). Specifically, if palatability-related activity observed in the "Late epoch" of GC taste responses truly depends upon BLA, then in- 
activation of BLA should preferentially affect these aspects, leaving earlier, "identity-related" aspects intact.

Here, we report the results of this experiment. Temporary inactivation of BLA (BLAx) halfway through GC recording sessions changed taste responses in all but a handful of GC neurons. BLAx affected firing rates across entire responses, but the impact was epoch-specific: Late-epoch palatability-related information was abolished or diminished in all but one small, identifiable subset of neurons, while identity-related information was left intact. Thus, BLA plays a powerful role in the driving of palatabilityrelated responses in GC, but the relationship between the two structures appears to be complex.

\section{Materials and Methods}

\section{Subjects}

Female Long-Evans rats ( $n=45 ; 275-300 \mathrm{~g}$ at time of surgery) served as subjects in this study. Animals were maintained on a $12 \mathrm{~h} \mathrm{light/dark}$ schedule, with experiments performed in the light portion of the cycle. Rats had ad libitum access to food and water. All methods comply with the Brandeis University Institutional Animal Care and Use Committee guidelines.

\section{Surgery}

Rats were anesthetized using an intraperitoneal (IP) injection of a ketamine/xylazine/acepromazine mixture (initial dose: 100, 10, and $10 \mathrm{mg} /$ $\mathrm{kg}$, respectively; maintenance: $1 / 3$ induction dose every $1.25 \mathrm{~h}$ ). The anesthetized rat was placed in a stereotaxic frame, its scalp excised, and holes bored in its skull for the insertion of self-tapping ground screws, electrode bundles, and guide cannulae for infusions. Bundles of 16 formvar-coated, $25 \mu \mathrm{m}$ nichrome wires attached to a mini-microdrive (Katz et al., 2001) were inserted $0.5 \mathrm{~mm}$ above GC (coordinates: anteroposterior (AP) $1.4 \mathrm{~mm}$ and mediolateral (ML) $\pm 5 \mathrm{~mm}$ from bregma; dorsoventral (DV) $-4.5 \mathrm{~mm}$ from dura). Guide cannulae were inserted into BLA (AP $-3 \mathrm{~mm}$ and $\mathrm{ML} \pm 5.1 \mathrm{~mm}$ from bregma; DV $-7.7 \mathrm{~mm}$ from skull) and stainless steel stylets were inserted into the guide cannulae to prevent blockage. Assemblies were cemented to the skull, along with two intraoral cannulae (IOCs; flexible plastic tubing inserted close to the tongue in the cheek and extending upward to the top of the skull) using dental acrylic. Rats were given $7 \mathrm{~d}$ to recover from the surgery.

\section{Water restriction}

Mild water restriction ( $45 \mathrm{~min}$ access at the same time every day), started $7 \mathrm{~d}$ following surgery, kept rats motivated and engaged in the task. Typically, $3 \mathrm{~d}$ are needed to train rats to drink their daily quotient of water in $45 \mathrm{~min}$. Daily records were kept of weight and water intake to ensure rats did not fall below $85 \%$ of normal values. The normal growth curves for these rats were downloaded from Charles River breeder's webpage and were kept with the daily records.

\section{Taste administration}

After beginning water restriction, rats were habituated for several days to the test chamber and allowed to move freely about the space for $\sim 1 \mathrm{~h}$ while receiving IOC deliveries of water to ensure calm, passive acceptance of pulsatile infusions. Testing sessions were identical to adaptation sessions, but with $40 \mu \mathrm{l}$ aliquots of (in $\mathrm{M}$ ) $0.1 \mathrm{NaCl}, 0.1$ sucrose, 0.2 citric acid, and 0.001 quinine- $\mathrm{HCl}$ delivered across $130( \pm 30) \mathrm{ms}$, instead of just water. Tastes were selected randomly without replacement, and each taste delivery was separated by a $40 \mu \mathrm{l}$ aliquot of water rinse (time between each fluid delivery was $10 \mathrm{~s}$ ). Sessions lasted $\sim 1 \mathrm{~h}$ (with drug or saline vehicle infusion after the first $30 \mathrm{~min}$ ), for total of $\sim 45$ trials of each taste per session. An additional 30 min session either 5 or $8 \mathrm{~h}$ postinfusion tested recovery from BLAx.

\section{Muscimol infusions}

Immediately following Intact sessions, rats were held in the experimenter's lap, and infusion cannulae were inserted into the previously implanted guide cannulae. Muscimol (100 ng/0.5 $\mu \mathrm{l}$; MP Biomedicals) or saline vehicle was infused bilaterally into BLA at a rate of $0.25 \mu \mathrm{l} / \mathrm{min}$ for $2 \mathrm{~min}$ (total infusate, $0.5 \mu \mathrm{l}$ ). This choice of muscimol concentration was based on a pilot study (for Wang et al., 2006) determining the highest concentration that preserved normal feeding behavior; similar concentrations have previously been found to impact learning but not naive behavior (Berlau and McGaugh, 2003; Müller and Fendt, 2006; Lee and Lim, 2010). A small set of pilot rats confirmed this lack of impact (data not shown).

Infusion cannulae were left in place for an additional minute after the pump stopped, to allow infusant to diffuse away from the tips of the guide cannulae. The functional spread of infusions delivered using this protocol was limited to $<1.5 \mathrm{~mm}$ (Floresco et al., 2006; Marquis et al., 2007; Moreira et al., 2007). This limited spread has been independently verified in our lab using fluorescent muscimol (Fortis-Santiago et al., 2010; Neseliler et al., 2011), and also functionally (Wang et al., 2006). Rats were then returned to the testing chamber for the BLAx (or vehicle control) session.

\section{Electrophysiology}

Neural signals were collected from GC during taste sampling. Differential recordings were fed into a parallel processor capable of digitizing up to 32 signals at $40 \mathrm{kHz}$ simultaneously (Plexon). Discriminable action potentials of no less than 3:1 signal-to-noise ratio were isolated on-line from each signal using an amplitude criterion in cooperation with a template algorithm (Nicolelis et al., 1997). Using these criteria, neurons were held through three distinct 30 min sessions, over the course of a $10 \mathrm{~h}$ day. Discriminations were checked continuously throughout each session. Time-stamped records of stimulus onset and neuronal spikes were saved digitally, as were all sampled spike waveforms and the discrimination file (Nicolelis et al., 1997). Off-line reanalysis incorporating threedimensional cluster-cutting techniques confirmed and corrected on-line discriminations. Using these techniques, we have previously described neural activity that, when analyzed using classic techniques, accords well with what has previously been known of GC single neurons (Katz et al., 2001; Fontanini and Katz, 2006).

Small groups $(7.8 \pm 4.4)$ of single neurons recorded within the same session are referred to below as "simultaneously recorded ensembles" or simply "ensembles."

\section{Single-neuron taste responses}

We subjected single-neuron data to a sequence of analyses designed to characterize aspects of GC neural responses to taste stimulation. Below we define these aspects and the analyses used to test them.

"Taste responsiveness." A neuron was deemed "taste-responsive" if its evoked firing rate in the $2.5 \mathrm{~s}$ poststimulation was significantly different from its baseline firing rate, evaluated via paired $t$ tests with significance level set to $p<0.001$. Note that a neuron could be taste-responsive despite responding identically to every taste; this analysis revealed only whether at least one taste caused firing to vary from steady, spontaneous rates.

"Taste specificity." A neuron's responses were deemed "tastespecific" in a manner akin to that used in visual cortex: neurons in V1 are "orientation-specific" if their responses vary with orientation, even if they respond broadly/ similarly to bars at a range of line orientations; we classified an individual neuron as taste specific if its responses significantly varied with taste, regardless to the specific breadth of that response. In neither modality is this definition intended to reveal a particular number of responses as distinct, but only that the response is nonuniform across the stimulus array. Taste specificity was evaluated by comparing the firing rate elicited by the 4 tastes, via two-way (time $\times$ taste) ANOVAs with poststimulus time divided into 10 consecutive 250 $\mathrm{ms}$ bins - a bin size small enough to allow a relatively fine-grained analysis and an approximate (but only approximate) division of epochs, but large enough to be relatively stable. A neuron was taste specific if either taste or taste $\times$ time factors were significant at $p<0.001$.

Note that, as this technique compared responses to each other rather than comparing each to baseline firing (the latter being an inappropriate method for assessing whether the neuron responded differentially to the tastes; Nieuwenhuis et al., 2011), neurons can be taste-specific without being taste-responsive, per se-for example, an inhibitory response and an excitatory response, each of which fails to be significantly different 
from baseline ( $p=0.1$, say), may well differ significantly from each other.

We further assessed taste specificity (see Fig. 4C) by subjecting each neural ensemble to a jackknife cross-validation classification test (Foffani and Moxon, 2004). Single trials of ensemble responses to a specific taste (from 250 to $1750 \mathrm{~ms}$ poststimulus divided into $250 \mathrm{~ms}$ bins) were compared with the average responses across all other trials grouped by taste (i.e., the single trial is "jackknifed"). We then calculated the Euclidean distance in $n$-dimensional space ( $n=$ the number of neurons) from each single trial to each taste template: if the minimum "distance" was to the same taste's template, the classification was deemed correct; abovechance (i.e., $>25 \%$ ) performance revealed taste specificity.

"Palatability relatedness." A neuron's responses were deemed "palatability-related" if they reflected the intrinsic agreeability/aversiveness of a taste. For this purpose we used several distinct but convergent analyses, each based on the well established preference relationships between the tastes (Grill and Norgren, 1978b; Breslin et al., 1992; Berridge, 2000).

First, we calculated the correlation coefficient $(r)$ between each neuron's firing rate responses to the battery of tastes and a simple linear palatability function [based on the above references: Sucrose (S) more palatable than $\mathrm{NaCl}(\mathrm{N})$, which is much more palatable than citric Acid (A), which is more palatable than Quinine (Q)]. The magnitude/significance of that correlation provided a measure of how well betweenstimulus differences in response were accounted for by palatability - that is, to what degree the responses were palatability-related.

Second, we computed a "palatability index" (PI) by comparing the similarity of single neurons' responses to tastes with similar palatability (i.e., S and N, Q, and A) to that for tastes with different palatability (S and $\mathrm{A}, \mathrm{S}$ and $\mathrm{Q}, \mathrm{N}$ and $\mathrm{A}, \mathrm{N}$ and $\mathrm{Q}$ ). The absolute differences in firing rates for each pair were averaged for pairs of the same type (similar or different). The PI consisted of the difference between these two types of differences (i.e., dissimilar - similar). Thus, an utter lack of palatability-related information results in a palatability index of 0 (because tastes of similar palatability and tastes of different palatabilities are equally different), and the more palatability-related the response, the more positive the PI. "Reverse palatability" - $S$ being more similar to $\mathrm{Q}$ than to $\mathrm{N}$, for instancedrives the PI to negative values. To avoid artificially attenuating the effect by including what appears to be a gradual emergence of the Late epoch (Fig. 1C; see Fig. 7A), the PI was calculated from firing in the "hearts" (i.e., not the onsets) of the epochs-200-600 ms postdelivery for the Middle epoch, and 1200-1700 postdelivery for the Late epoch. Results were similar, however, for a range of sampling periods (data not shown).

Third, we used an error analysis of the above-described classification task to further compare palatability-related content of Intact and BLAx taste coding. In neurons responding in a "palatability-neutral" manner, errors should be random; in neurons for which responses are palatabilityrelated, however, the classification task should more commonly misattribute a specific single-trial response for a specific taste to the other taste of the same palatability than to tastes of different palatability-S trials should more often be misidentified as $\mathrm{N}$ trials than as $\mathrm{Q}$ or A trials, for instance. We therefore used a $\chi^{2}$ analysis to compare the pattern of errors produced when the classification task was applied to the Late epochs of either Intact or BLAx sessions, testing the hypothesis that the pattern of errors should become more uniform in the latter sessions if in fact BLAx reduces the palatability specificity of GC responses.

Note that palatability-relatedness assumes taste specificity; a neuron that is the former is necessarily the latter.

\section{Changes in GC taste responses across poststimulus time}

To explicitly examine how each aspect of the GC neural responses changed across time, the methods described above for examining taste responsiveness, taste specificity, and palatability relatedness were repeated using a moving window: the window, which was set to $200 \mathrm{~ms}$, was advanced by $50 \mathrm{~ms}$ steps, and the significance level was set to 0.001 ; for the moving window tests of "taste specificity," the two-way ANOVA was replaced with 1-way ANOVA with taste as a single factor.

As described above, we also performed analyses for which firing rates were averaged across time ranges within the hearts of epochs. Statistical comparisons of these averages (via $t$ tests or ANOVAs) revealed epochspecific effects (to ensure lack of bias in such tests, care was taken to ensure that the same amount of time was included in the averages for each epoch).

In all cases described above, analyses were extended to comparisons of Intact and BLAx responses.

\section{Change point analysis}

Transitions in the effect of BLAx on ensemble firing rates following taste deliveries were detected with a technique making use of the cumulative sum of deviations from the mean to detect changes in ordered values (Hinkley, 1971). First, the ensemble firing rate differences were binned in consecutive $250 \mathrm{~ms}$ segments, and a $\chi^{2}$ test (described above) was used to determine the non-stationarity of the distribution. The rates were then summed, and the mean was subtracted to produce a series of normalized deviations from that mean. The single most likely change point was then defined for each ensemble as the point with the maximum absolute difference.

\section{Histology}

At the conclusion of the experiment, rats were deeply anesthetized and perfused transcardially with $0.9 \%$ saline followed by $10 \%$ formalin. Seven seconds of DC current $(7 \mu \mathrm{A})$ were passed through selected microwires in preparation for staining. Brains were removed and refrigerated for several days in 30\% sucrose/ 10\% formalin. Coronal sections (60 $\mu \mathrm{m})$ were cut using a freezing microtome and mounted to glass slides. Sections were stained with cresyl violet (cell bodies; Sigma-Aldrich) and Prussian blue (ferrous deposits blasted off electrode tips) to visualize cannulae and electrode tracks respectively. From these sections, cannula tip locations were marked on the appropriate brain region coordinates (Paxinos and Watson, 2007). The distance of these tips from the center of either the BLA or GC along a horizontal line was measured at the dorsalventral level of each tip site (as described by Wang et al., 2006). The medial-lateral extent of the region was measured and bisected, and the distance of the tip mark from that bisection point was recorded. As noted above, muscimol infusions were limited to $1.5 \mathrm{~mm}$, and only data from animals with electrodes (GC) and cannulae (BLA) placed within this infusion limit were included in the analyses.

\section{Results}

\section{Taste responses in GC}

Once adapted to the testing paradigm, rats underwent the entire experimental procedure across the course of a single 7-10 h day (see Materials and Methods; Fig. 1A). Each of three recording/ tasting sessions lasted $30 \mathrm{~min}$, with a $10 \mathrm{~min}$ muscimol infusion interpolated between the first two ("Intact" and "BLAx") sessions. Either 5 or $8 \mathrm{~h}$ postinfusion, rats underwent the final 30 min recording/ tasting "Recovery" session, identical to both of the previous sessions (rats were returned to their home cages for the interval between BLAx and Recovery sessions).

A total of 145 GC neurons, collected from chronically implanted electrode bundles in 19 rats, were successfully held across the entire protocol (see Fig. 5, $7.8 \pm 4.4$ neurons/ rat; example waveforms for neurons with representative taste responses are shown). Multiple control datasets were collected as well: 19 rats underwent an identical experimental protocol but with saline vehicle infused instead of muscimol (95 neurons), and another 4 rats had muscimol infused into the nearby central nucleus of the amygdala (CeA; 27 neurons); neurons recorded from rats in which one cannula missed BLA (confirmed by histology, Fig. 1C) served as a third control.

GC neuronal responses to $\mathrm{NaCl}$, sucrose, citric acid, and quinine recorded in the Intact session were consistent with those observed previously in multiple labs (Yamamoto et al., 1985; Katz et al., 2001; Bahar et al., 2004; Jones et al., 2007). Of the 145 neurons recorded, 89 were taste-responsive-i.e., their firing rates differed significantly from baseline following taste delivery. 
Furthermore, many neurons responded in a taste-specific manner, producing distinct magnitudes or time courses of neural firing to different tastes. Using a conservative criterion $(p<0.001$ in either the main effect for taste or the taste $\times$ time interaction of a two-way ANOVA; see Materials and Methods), 67 of the 89 taste-responsive neurons, and 85 (58.6\%) of the total 145 sampled GC neurons, were deemed "taste-specific." These percentages, and those observed in analyses of smaller amounts of data (single epochs, see below), accord well with our own previous work, and with most of the large literature on primary cortical sensory responses. For $55.3 \%$ of the taste-specific subsample (i.e., $32.4 \%$ of the overall sample, $n=47)$, differences in the time courses of the responses accounted for the taste specificity (i.e., significant taste $X$ time interactions).

The time-varying responses were themselves similar to those observed in previous studies (Katz et al., 2001; Fontanini and Katz, 2006; Grossman et al., 2008). Figure 2 shows two examples of these responses. Figure $2 C$ presents moving window analyses of taste-responsiveness, taste specificity, and palatability-relatedness for the entire Intact dataset (see Materials and Methods for definitions and descriptions of the analyses). These analyses confirm: (1) that taste-responsiveness appeared immediately, peaked within the first $100 \mathrm{~ms}$, and declined afterward; (2) that taste specificity was largely absent during this earliest aspect of the response (the "Early epoch"), instead developing across the first few hundred ms of poststimulus firing (the "Middle epoch"); and (3) that the number of neurons producing palatabilityspecific responses rose still more slowly, becoming significant ( $p<0.01$ by $t$ test, compared with baseline) only in the last half of the first second (i.e., the approximate onset of the "Late epoch," see Discussion).

Thus, as with previous observations, GC firing rates change immediately when tastes cross the tongue, become taste-specific within $\sim 200 \mathrm{~ms}$, and become palatability-specific after another several hundred milliseconds. The emergence of palatabilityrelated firing is associated with a relatively modest decrease in the number of neurons responding to each taste (Fig. 2D) and in overall firing rates (see below), and with an attendant shift toward narrower "response tuning" (that is, fewer significant taste responses per neuron, Fig. 2 E). We observed no increase, however, in the likelihood of neurons to respond to two and only two tastes in the Late epoch; thus, "palatability coding" is not simply reflected a tendency of GC neurons to respond significantly to only two (same-palatability) tastes, as it appears to be for BLA neurons (Fontanini et al., 2009).

Note that within the reliable framework described above, response time courses to individual tastes vary from neuron to neuron (Erickson et al., 1994; Katz et al., 2001; Bahar et al., 2004; Jones et al., 2007). Even when a pair of simultaneously recorded neurons responded to some of the same tastes (such as the neurons contributing the responses shown in Fig. $2 A, B$ ), there was no canonical "sucrose response" (or "quinine response," etc) that was reliably observed across multiple neurons.

\section{BLAx changes but does not eliminate GC taste responses}

BLAx affected the taste responses of GC neurons, and did so in one of two distinct ways. Figure $3, A$ and $B$, show representative examples of the two types of observed changes. The neuron in Figure $3 A$ shows what proved to be the modal effect, ubiquitous reductions of response magnitudes. Note that BLAx did not actually obliterate this GC cell's ability to fire-in fact, the neuron continued to respond distinctly to different tastes following inactivation of BLA, with a response to citric acid that was more than twice the magnitude of its response to $\mathrm{NaCl}$ - but it reduced each response's magnitude. Also observed (although more rarely) were BLAx-induced response enhancements, which appeared to include and even introduce taste specificity (Fig. 3B; this subset of neurons will be discussed below). Note, however, that taste specificity, absent in the Early epoch of the Intact responses, remained so during BLAx despite the response enhancement and unmasking.

Across the entire GC dataset, this sort of BLAx impact could be observed in 142 (98\% of 145) neurons (Fig. $4 A$; percentages were nearly identical regardless of whether analyses were brought to bear on neurons that were taste-specific, taste-responsive, or both). When BLAx affected any of a neuron's taste responses, it typically affected most of these responses, and typically changed firing rates across the entirety of the response to at least some degree (although not uniformly, see below). In fact, in 65\% ( $n=$ 94) of the neurons affected, BLAx altered responses to every stim- 
A

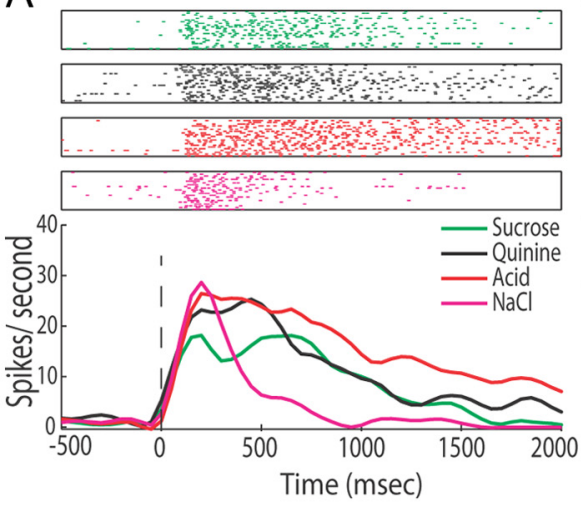

B
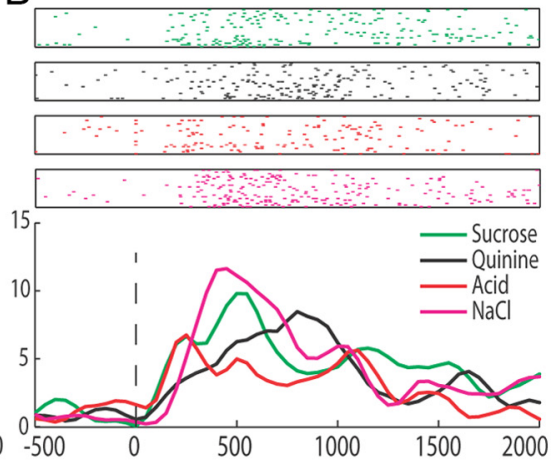

C

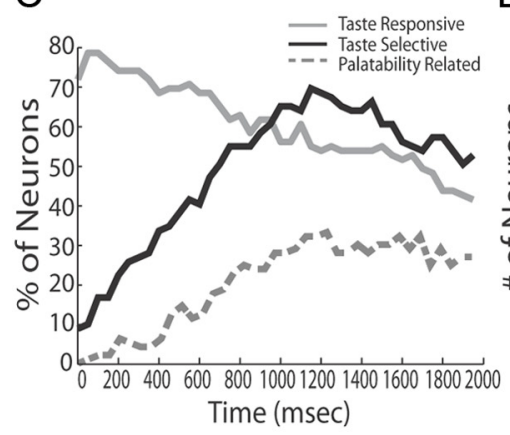

D

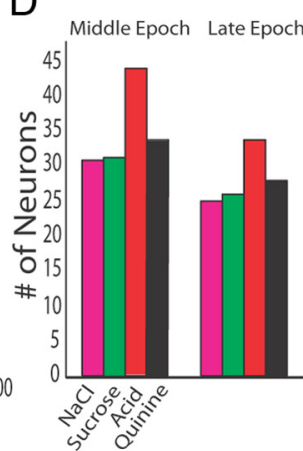

$\mathrm{E}$

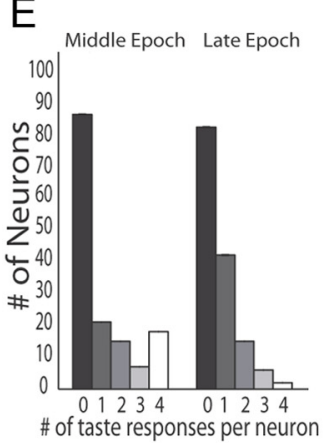

Figure 2. Taste responses can be observed in $\mathrm{GC} . A$, The top half shows raster plots of spiking activity of a single representative $\mathrm{GC}$ neuron in response to presentation of the four basic tastes (sucrose, green; quinine, black; citric acid, red; $\mathrm{NaCl}$, pink). Each row is a single trial, and each dot is an action potential. Below are the corresponding PSTHs, which show the firing rate in spikes/second ( $y$-axis) of the neuron's response to taste; $x$-axis, poststimulus time in milliseconds. Zero is the moment of taste delivery, marked with a vertical dashed line. $B$, A second representative neuron's response to the same tastes. $C$, The percentage of GC neurons ( $y$-axis) that are taste-responsive (gray), taste-selective (black), and palatability-related (dashed) when BLA is intact ( $x$-axis, poststimulus time in ms). Because each trace reflects an analysis involving a sliding window, some smoothing has necessarily occurred. $\boldsymbol{D}$, The number of neurons ( $y$-axis) responding to each of the four tastes ( $x$-axis) during the Intact Middle and Late epochs; somewhat fewer neurons respond to each taste in the Late epoch. $E$, The number of neurons ( $y$-axis) that responded to a particular number of taste stimuli $(x$-axis) during the Intact Middle and Late epochs.

A
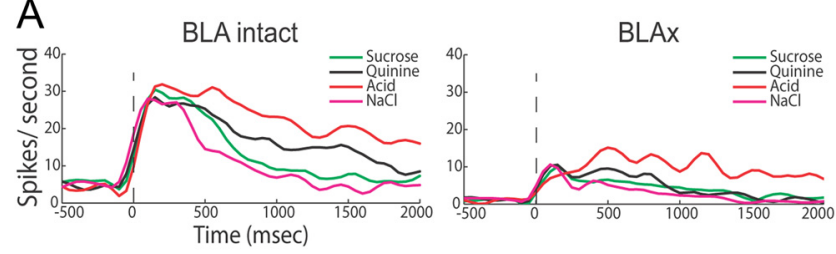

B
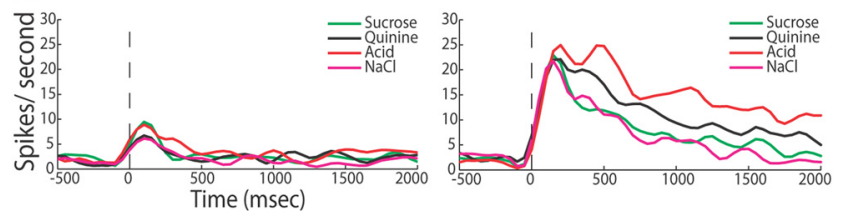

Figure 3. BLAx changes firing rate of responses. $\boldsymbol{A}$, Representative example of how a GC neuron's responses to tastes in the Intact session (left PSTHs) could be altered following muscimol infusion into BLA - in this case, BLAx ubiquitously decreased responses (right PSTHs). Same conventions as Figure 2. B, A second example GC neuron's responses, showing (less common) firing-rate increases wrought by BLAx.

ulus; in another $28 \%(n=41)$ of the neurons, responses to either 2 or 3 (of the 4) stimuli changed. In all, a full $71 \%$ of the 580 individual assayed taste responses ( 145 neurons $\times 4$ tastes) were changed by BLAx, and the distribution of number of taste responses changed per neuron by BLAx was decidedly skewed to- ward more responses changed (Fig. $4 A$, $\chi^{2}(3)=64.93, p<0.001$ in a comparison of the observed and a uniform distribution). Furthermore, the effect of BLAx on any particular neuron's responses tended to be in a uniform direction: for $86 \%$ of the neurons affected $(n=122)$, all responses changed in the same direction (e.g., either all suppressed or all enhanced).

Control infusions changed relatively few GC taste responses. Vehicle infusions, for instance, affected a subset of responses in only $11 \%$ of the GC neurons (i.e., $2.5 \%$ of the assayed responses, a level of impact approximating chance). Of the 17 neurons recorded from rats in which one of two cannulae missed BLA, meanwhile, $71 \%(n=12)$ maintained all of their original responses to tastes following muscimol infusions (i.e., a subset of responses changed in 29\% when one BLA was inactivated), a level of impact appropriately suggestive of incomplete BLAx. Finally, muscimol infused into the CeA, the tasteresponsive site nearest to BLA, changed responses in 33\% of GC neurons ( $n=9$ of 27 neurons), and changed $33 \%$ of the individual taste responses (32 of 108, data not shown). While this last result confirms an involvement of CeA in taste coding (see Discussion), our bilateral BLA-centered infusions changed three times the number of responses affected by $\mathrm{CeA}$ inactivation (CeAx). Thus, the larger impact of BLAx is almost certainly the result of our inactivation of BLA neurons, and neither the result of muscimol infusion itself nor of incidental inactivation of neurons in other regions. We therefore performed no further analyses on the control datasets. GC taste responses are exquisitely sensitive to the presence or absence of BLA, despite the continued patency of the thalamocortical taste pathways.

The most likely specific impact of BLAx on a single neuron was an at least partial suppression of all responses (19 neurons became entirely unresponsive with BLAx), and the second most likely impact was enhancement of all taste responses ( 15 neurons became taste neurons because of BLAx). Of the neurons in which all responses were changed to some degree, $62.1 \%$ underwent response suppression, more than underwent response enhancement $\left(\chi^{2}(1)=15.79, p<0.001\right)$. The overall impact, in analysis of both Middle and Late epochs, was a significant but far from complete (and again, far from uniform across the time course of the responses, see below) decrease in the average firing rate of GC neurons in response to taste administration (Fig. 4B).

In addition to being on average moderate in magnitude, the changes wrought by BLAx did not deprive GC of its ability to respond distinctively to different taste stimuli. Of 145 neurons, $59.3 \%(n=86)$ responded in a taste-specific manner $(p<0.001$ in either the main effect for taste of the taste $\times$ time interaction of a two-way ANOVA applied to the first $1.75 \mathrm{~s}$ of the responses) during BLAx; of these, $54.7 \%$ ( $n=47,32.4 \%$ of the total sample) produced taste-specific time-varying responses (significant 
taste $\times$ time interaction). These numbers are comparable to those observed for the same neurons in the Intact session.

Figure $4 C$ presents a deeper examination of this finding, showing the result of a peristimulus time histogram (PSTH) classification algorithm brought to bear on ensemble-by-ensemble responses in both Intact and BLAx sessions (see Materials and Methods for details). This analysis confirmed both that GC responses were reliably taste-specific in the Intact session-the delivered stimulus could be correctly identified in $59.5 \%$ of the trials of each taste (chance $=25 \%$ ) - and that similar performance was maintained in the BLAx session (57\% correct identification, no significant difference between the Intact and BLAx sessions, $t_{(75)}=0.608$; $p>0.5)$. That is, taste-specific information remained readily available in GC following BLAx, despite massive changes to specific responses. This result held for any analysis that included the Middle or Late epochs of the responses.

\section{GC taste responses recover following BLAx}

Direct measurements have suggested that neurons in awake animals recover from muscimol-induced inactivation after several hours (Martin and Ghez, 1999; Arikan et al., 2002; van Duuren et al., 2007). Our Recovery data are consistent with these conclusions. Representative example neurons are shown in Figure 5, $A$ and $B$. One of these neurons experienced BLAxinduced response suppression, and the other response enhancement. In both cases, the preinactivation responses largely recovered within $8 \mathrm{~h}$. Of the $71 \%$ (of 580) individual GC neural responses that were significantly altered by BLAx, only $40 \%$ had recovered their preinactivation firing rates within 5 h; by 8 h postinfusion, $64 \%$ had recovered, suggesting that recovery was in progress, but far from complete, by this time.

\section{BLAx reduces Late epoch evidence of} palatability processing, despite sparing taste specificity In our previous work, we characterized the stimulus-specific portion of GC taste responses (recorded as in the current preparation) as consisting of a 2-epoch sequence following a brief epoch of nonspecific response. Responses in the first of these two tastespecific epochs (i.e., the Middle epoch) thus far appear to reflect mainly taste identity, whereas in the Late epoch responses cluster according to taste palatability. The transition between these two epochs has been shown in multiple studies to occur late in the first second of the taste response (Katz et al., 2001; Fontanini and Katz, 2006; Grossman et al., 2008), although estimates of the actual transition time necessarily varies somewhat from experiment to experiment, and even from trial to trial (Jones et al., recovered within $8 \mathrm{~h}$.
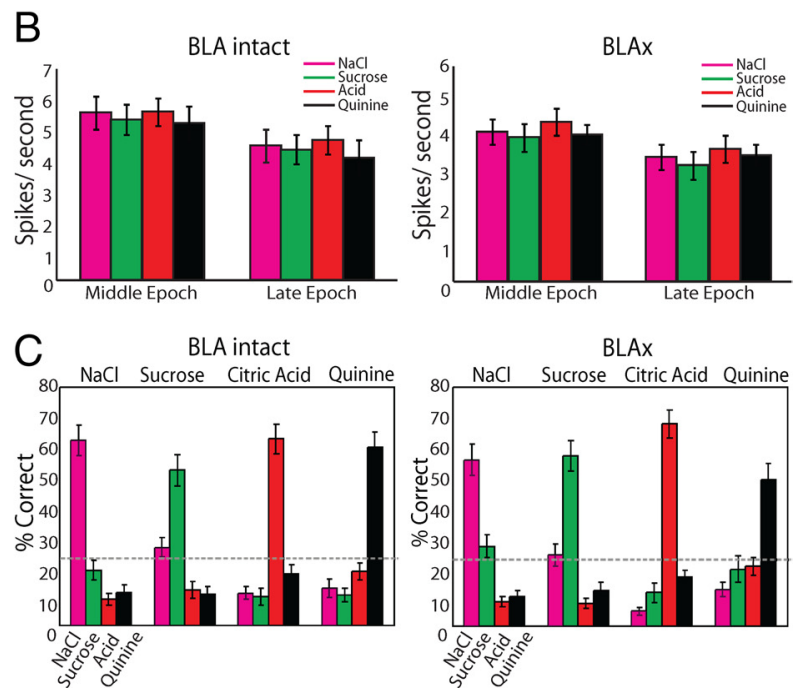

Figure 4. The overall impact of BLAx on the entire $\mathrm{GC}$ population. $\boldsymbol{A}$, Pie chart showing how different $\mathrm{GC}$ neurons were affected by BLAx. For $65 \%$ of the neurons, responses to all 4 tastes changed (blue); for $55.9 \%$ of these, responses were either all decreased $2 \%$, dark blue) or all increased (17.7\%, light blue; hatching shows the few single neurons for which some changes were reductions and others enhancements). BLAx changed responses to 3 of 4 tastes for $12 \%$ of the remaining GC neurons (purple), changed responses to 2 tastes in 16\% (green), etc. $\boldsymbol{B}$, Graph showing the firing rate in spikes/second ( $y$-axis) for all tastes in the rates between the Middle and Late epochs within a session was significant ( $p<0.05$ by $t$ test). The difference in firing rate within a single taste was likewise significant ( $p<0.05$ by $t$ test) within an epoch across sessions. $C$, The result of a classification analysis, istered taste (top) — was similarly high in Intact (left) and BLAx (right) sessions, across the overall GC population. Along the $x$-axis
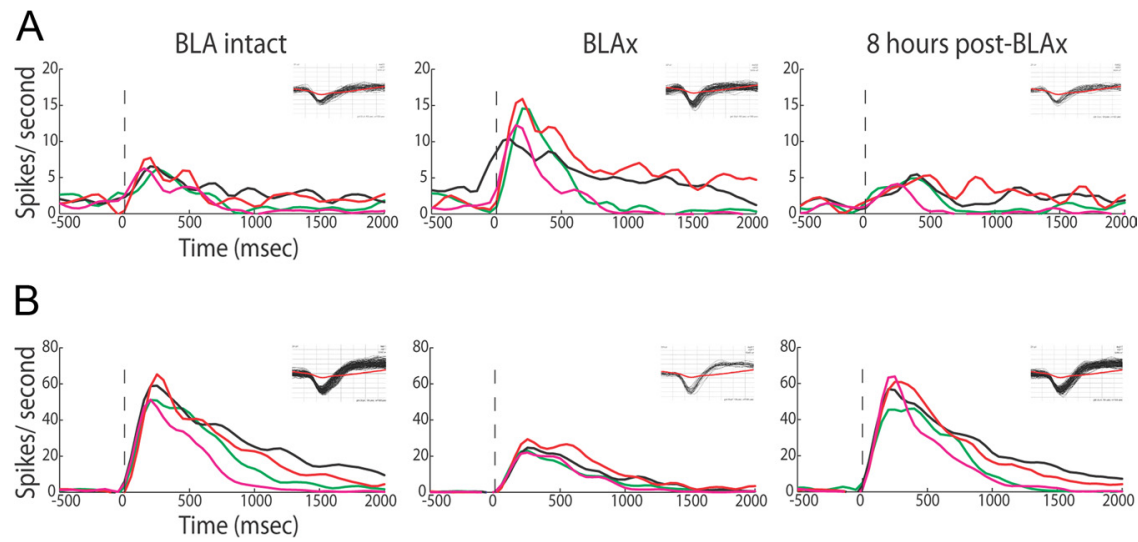

Figure 5. Taste responses recover within $8 \mathrm{~h}$ after muscimol infusion. $\boldsymbol{A}, \mathrm{PSTH}$ (same conventions) for a representative GC neuron, showing responses increasing following BLAx and recovering within $8 \mathrm{~h}$; the insets show corresponding waveforms (black) with average noise trace (red). $\boldsymbol{B}$, PSTH of second representative neuron for which responses decreased following BLAx and

2007). In BLA, meanwhile, palatability-related firing appears in the Middle epoch (Grossman et al., 2008; Fontanini et al., 2009). This fact, in conjunction with a wealth of data collected by other researchers (Schoenbaum et al., 1998; Bechara et al., 1999, 2003), led us to propose that BLA might be a "source" of palatability responses in cortex, and more specifically to predict that the primary effect that BLAx would have on the information available in taste-related spiking would be to reduce the amount of palatability specificity found in Late-epoch GC responses.

The simplest version of this hypothesis posits that BLAx should only change firing rates in this Late epoch, leaving earlier portions of the responses completely unchanged. Figures 3 and 5 clearly demonstrate that this simplistic hypothesis does not hold. 
A
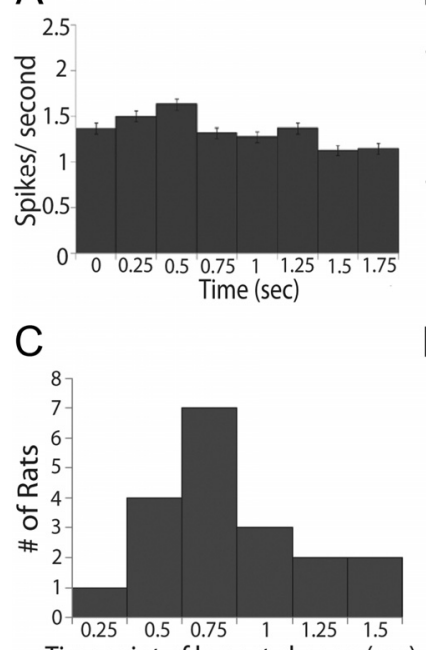

Timepoint of largest change (sec)
B

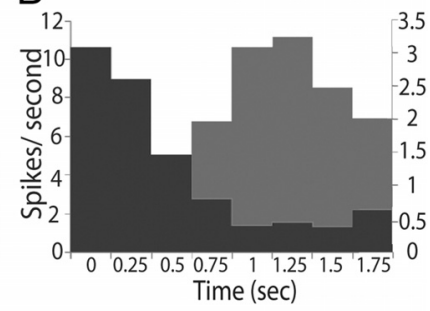

D

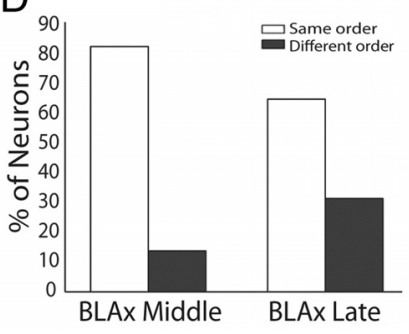

Figure 6. Epoch dependency of the effects of BLAx. $\boldsymbol{A}$, Absolute differences in firing rates (spikes/s, $y$-axis) between Intact and BLAx sessions for the entire sample ( $n=145$ ) of GC neurons across time in seconds ( $x$-axis), divided into $250 \mathrm{~ms}$ bins. $\boldsymbol{B}$, Similar histograms for representative ensembles from two animals demonstrating time-structure that is lost in the overall average; for one ensemble (dark gray), BLAx-induced changes measured in terms of absolute differences in firing rates were high early and lower late; for the other example (light gray), the pattern was reversed. The $x$-and $y$-axes are the same as for $\boldsymbol{A}$. $\boldsymbol{C}$, The results of change point analysis brought to bear on the data for each ensemble, showing that the impact of $B L A x$ reliably changed between 0.75 and $1 \mathrm{~s}$ (bins 3 and 4 ). $y$-axis, Number of ensembles for which the greatest change in impact of BLAx occurred between particular pairs of bins; $x$-axis, time point of the largest change in seconds. $\boldsymbol{D}$, The percentage of neurons ( $y$-axis) where the order of responsiveness (e.g., which tastes caused the largest, second largest, third largest, and smallest responses) was the same (open) during BLAx as BLA Intact or different (gray) in both the Middle and Late epochs ( $x$-axis).

A related but more subtle prediction was borne out, however: the impact of BLAx on GC responses proved to be distinct for Middle and Late epoch responses, such that BLAx reduced the palatability-related content of Late epoch firing despite leaving taste specificity intact.

Initial evidence for this conclusion comes from an analysis demonstrating that the impact of BLAx on firing rates differed for Middle and Late epochs. Averaged across the entire dataset, the effect of BLAx on firing rates was largely uniform across time (Fig. $6 A$ ), but this uniformity proved to be an artifact of averaging across neurons and ensembles: at the single-ensemble level, BLAx affected Middle- and Late-epoch firing quite differently; the apparent non-effect shown in Figure $6 \mathrm{~A}$ arose because for some ensembles changes were larger (in absolute magnitude) in the Middle epoch, while for others the pattern was reversed (examples are shown in Fig. $6 B$ ). $\chi^{2}$ goodness of fit tests demonstrated that for 17 of 18 sessions, within-animal distributions of firing rate changes were significantly nonuniform across time $(p<0.01)$.

In both cases shown in Figure $6 B$, the impact of BLAx appeared to change around the time of the transition between the previously identified taste-specific epochs-in one case becoming larger later, and in the other case becoming smaller. We statistically evaluated the phenomenon suggested in these examples by bringing a "change point analysis" to bear on every ensemble (see Materials and Methods for details). The summary of this analysis-presented in Figure $6 \mathrm{C}$-reveals that the impact of BLAx on GC neural activity typically changed after the bin ending $0.75 \mathrm{~s}$ post-taste delivery but before the end of the $0.75-1.0 \mathrm{~s}$ bin.

This significant result $\left(\chi^{2}(7)=1100.38, p<0.001\right)$ confirms our prediction that the impact of BLAx changes at a time point that coincides roughly with the onset of the Late epoch (Katz et al., 2001; Fontanini and Katz, 2006; Grossman et al., 2008).

While the above analysis demonstrates that BLAx-related changes of GC responses are distinct for the Middle and Late coding epochs, further analysis was required to reveal precisely how the information contained within those response epochs are affected by BLAx. Figure $4 C$ shows that taste specificity in GC largely survives BLA. We therefore next moved to directly testing our most central hypothesis - that is, determining whether BLAx deprived GC of palatability-related information.

A basic analysis of spike rates demonstrated that BLAx was much more likely to change the order of responsiveness-changing which taste caused the largest response, second largest response, etc. - in the Late epoch than in the Middle epoch (Fig. $\left.6 D, \chi_{\text {Middle vs Late }}^{2}(3)=4.6, p<0.03\right)$. A look at the example in Figure $3 A$ further suggests that BLAx specifically reduces the availability of palatability-related information in some neurons (exceptions such as the neuron shown in Fig. $3 B$ will be discussed below): in the Intact session, this neuron's responses had a palatability-related component (the sucrose and $\mathrm{NaCl}$ responses were relatively similar, as were the quinine and citric acid responses) that emerged across the period between 0.75 and $1.0 \mathrm{~s}$ after taste delivery; in the BLAx session this palatability-related patterning had vanished, even though the responses remained taste-specific.

Examination of the entire dataset confirmed the representativeness of this example. A moving window analysis of how well each neuron's responses correlated with the known palatability of the taste stimuli in Intact sessions (as determined in many studies, see Materials and Methods) showed, as expected, that palatability-related information grew slowly across the first second of the responses, peaking at $\sim 1 \mathrm{~s}$ after stimulus delivery (Fig. $7 A$; see also Fig. 2C). The same analysis of BLAx session data largely overlays the Intact session data for $\sim 600 \mathrm{~ms}$, after which the two lines diverge- at approximately the time of the onset of the Late epoch. The difference becomes significant (paired $t$ tests with $\mathrm{df}=147)$ at $\sim 1.1 \mathrm{~s}$ into the responses. Thus, there is less palatability-related information in the Late-epoch GC responses during BLAx.

Independent confirmation of this result came from a PI comparing the relative differences of each neuron's response to tastes of similar (i.e., sucrose- $\mathrm{NaCl}$ and quinine-acid) and different palatabilities (i.e., sucrose-quinine, sucrose-acid, NaCl-quinine, and $\mathrm{NaCl}$-acid; see Materials and Methods). Calculated in this manner, a positive PI reveals palatability-related responses, in that responses to tastes of like palatability are more similar than responses to tastes of different palatabilities.

We performed the analysis separately for the Middle and Late epochs, and for Intact, BLAx and Recovery sessions. Figure $7 B$ shows the results of this analysis, which were subjected to statistical evaluation by repeated-measures ANOVA (note that the ANOVA was brought to bear only on the Intact and BLAx sessions, because there was some loss of neurons between the BLAx and Recovery sessions). The ANOVA revealed main effects of session $\left(F_{(1,95)}=4.89, p<0.03\right)$ and epoch $\left(F_{(1,95)}=27.23, p<\right.$ $0.001)$, as well as a significant interaction between the two $\left(F_{(1,95)}=5.15, p<0.03\right)$; the interaction reflected a significant Late-epoch difference between Intact (light gray bar) and BLAx (dark gray bar, $t_{(95)}=2.44, p<0.02$ ). The Middle epoch showed no such Intact-BLAx difference; in fact, there was little or no palatability-related content in Middle epoch responses according 

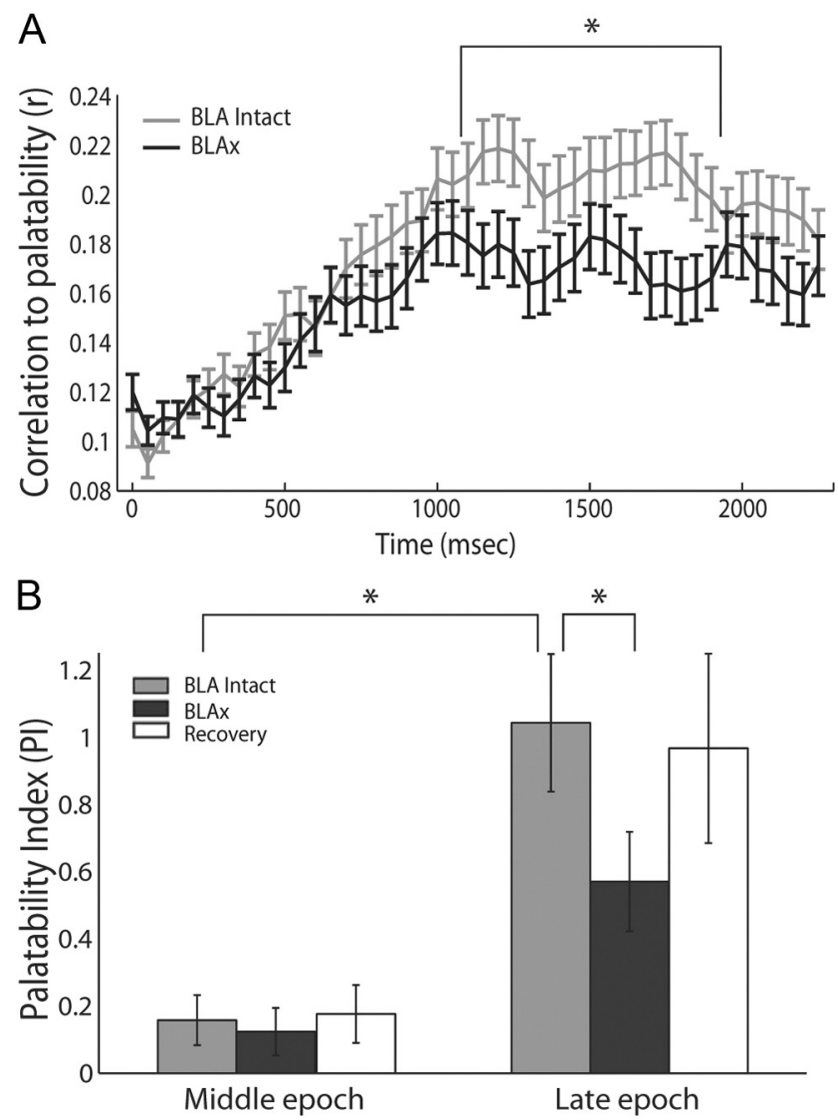

Figure 7. Late-epoch palatability processing is specifically affected by BLAx. A, Movingwindow analysis of the correlation between firing rates and taste palatability ( $y$-axis) across poststimulus time ( $x$-axis), performed separately for Intact (light gray) and BLAx (dark gray) sessions. As expected, the correlations rise to a peak only at the end of the first second (movingwindow procedure "smoothes" the data such that the correlation appears to rise earlier than it truly does). Note that the functions for Intact and BLAx diverge in the last half of the first second; the palatability correlation is significantly lower for BLAx starting at $1.1 \mathrm{~s}$. B, Summary of this change in palatability processing, using a PI ( $y$-axis) defined as the difference between the mean firing rate responses to tastes with similar and different palatabilities, computed separately for Intact (light gray), BLAx (dark gray), and Recovery (open) sessions. Note that the actual amount of palatability-related activity is close to zero (i.e., no palatability-related response) in the Middle epoch. The expected difference in palatability content between Middle and Late epochs during Intact sessions (light gray) is significant, as is the comparison between the Intact and BLAx Late epoch PI. For both panels, error bars represent SEM and ${ }^{*} p<0.05$.

to this analysis (this is not inconsistent with Fig. $7 A$, which, as a moving window analysis, under-estimates the latency at which information appears). The Late-epoch PI was in the process of returning to Intact levels by the time of the Recovery session (Fig. $7 B$, open bar) as expected.

Finally, we also examined errors made in the classification analysis described previously (Fig. $4 C$ ). While a change in palatability content does not necessarily predict a change in the rate of correct classifications, it does predict a subtle shift in the nature of errors made: specifically, if neurons are responding in a palatability-related manner, errors in classification should primarily misidentify a taste as the other taste of the same palatability (Fontanini et al., 2009); if responses are not palatability-related, meanwhile, errors should be random. We analyzed Late-epoch Intact session data, and found while few errors were made (correct classifications were made in nearly $60 \%$ of the trials), errors made using Late-epoch data from Intact sessions were twice as likely to be "withinpalatability" as "between-palatability." Meanwhile, in BLAx Late epoch data, the percentage of between-palatability errors (e.g., incorrectly classifying a sucrose trial as quinine) increased by $25 \%$, despite the fact that $>50 \%$ of the trials were identified correctly. This change, a third measure indicating a decrease in palatability-related information, was significant $\left(\chi^{2}(3)=17.3, p<0.001\right)$.

Thus, across a range of analyses, BLAx was specifically shown to reduce the prevalence of palatability-related activity in Lateepoch GC taste responses.

\section{BLAx unmasks a small sample of neurons receiving palatability-related information from elsewhere}

While the amygdala clearly plays an important role in selecting specific and experience-dependent palatability-related behaviors (Galaverna et al., 1993; Touzani et al., 1997; Nishijo et al., 1998), the brainstem is capable of driving basic taste reactivity (Di Lorenzo et al., 2009; Rosen et al., 2011), even in decerebrate rats (Grill and Norgren, 1978b). Furthermore, brainstem lesions can change taste palatability (Spector, 1995). Thus, it is to be expected that at least some palatability-related neural activity in GC should be independent of BLA, instead being a reflection of palatabilityrelated information processed in the brainstem itself.

In fact, we observed that one specific subgroup of GC neurons - identified before any analysis of palatability - failed to fit the overall pattern: while the neural sample as a whole was deprived of palatability-related responses by BLAx, there were some neurons in which taste responses were significantly enhanced by BLAx $(n=23,27 \%$ of taste-specific neurons and $15.9 \%$ of the total sample), and these enhanced responses appeared to contain Late-epoch palatability-related information. Figure $3 B$ shows an example of this phenomenon.

To test the generality of this phenomenon, we divided the dataset into two groups according to whether BLAx reduced or enhanced firing (a separation performed blind to palatability content). This analysis revealed that the entirety of the effect shown in Figure 7 was carried by the plurality of neurons for which BLAx reduced firing rates (Fig. $8 A$ ); a two-way ANOVA of these data revealed effects for epoch $\left(F_{(1,75)}=12.6, p<0.001\right)$ and session $\left(F_{(1,75)}=11.9, p<0.002\right)$ and the expected epoch $\times$ session interaction $\left(F_{(1,75)}=6.4, p<0.02\right)$. The interaction reflected the lack of Late-epoch palatability in the BLAx session $\left(t_{(75)}=3.18, p<0.005\right)$. Note that this severe reduction in PI was achieved despite the fact that many of these neurons continued to respond to tastes.

Meanwhile, neurons in which BLAx enhanced responses showed no discernible BLAx-related decrement of the PI-in the two-way ANOVA there was a significant main effect for epoch $\left(F_{(1,21)}=21.1, p<0.001\right)$ but no epoch $\times$ session interaction $\left(F_{(1,21)}<1, p>0.75\right)$. These neurons, which we suspect contain information processed in brainstem and passed to GC directly via thalamus (i e., not by some parallel path; see Discussion), drive what little palatability-related information can be seen in the entire BLAx sample in Figure 7.

\section{Discussion}

Although there is extensive evidence suggesting the importance of both amygdala and cortex in both taste perception and learning (Escobar et al., 1998a,b; Ferreira et al., 2005; Grossman et al., 2008; Fontanini et al., 2009), the only studies to have directly investigated the dependence of activity in one structure on the other have looked at very different cortical regions than the one we study here, and for very different aims (Paré et al., 2002; Saddoris et al., 2005; Bauer et al., 2007; Popa et al., 2010).

Our own recent data have indirectly suggested cooperative coding of taste between the GC and the BLA. Neurons in the two 


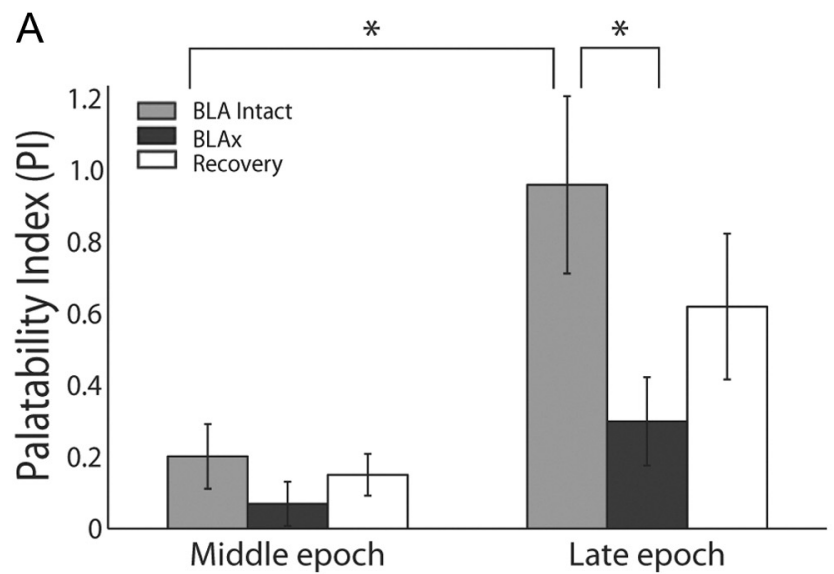

B

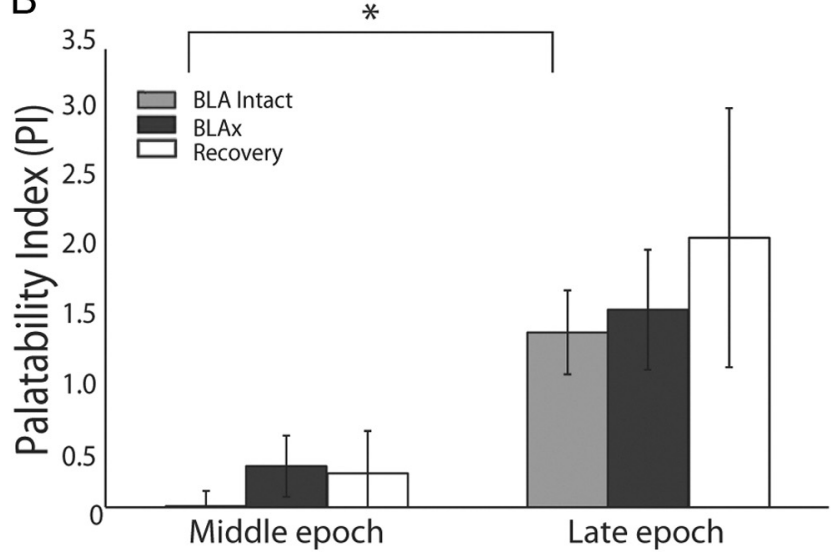

Figure 8. Palatability processing is not affected by neurons with increasing firing rate postBLAx. A, PI analysis (conventions same as Fig. 7) for the specific subgroup of neurons for which response magnitudes were reduced by BLAx. The growth in palatability content from the Middle to the Late epoch is significant for Intact sessions, but not for BLAx sessions. Within the Late epoch, there is a significant difference between Intact and BLAx. $\boldsymbol{B}$, When analysis is restricted to the subgroup of neurons for which responses are enhanced $B L A x$, the significant palatabilityrelated difference between Intact and BLAx sessions in the Late epoch disappears. Note the difference in $y$-axis scaling for the two panels. ${ }^{*} p<0.05$.

regions progress through synchronous rate transitions, but palatability-related information appears in BLA one "epoch" earlier than it does in GC (Fontanini et al., 2009). While these data provide support for the hypothesis that emotional labels may be attached to stimuli (at least in part) by BLA before being used in cooperation with cortex (Schoenbaum et al., 1998; Bechara et al., 1999, 2003; Popa et al., 2010) — a hypothesis that is also supported by the fact that functional BLA-GC connectivity is increased during emotional learning (Grossman et al., 2008) — they do so only indirectly.

In the realm of taste, the most direct prediction of the above hypothesis is that BLAx should reduce palatability-related content - content that normally appears in the latter half of the first second following taste delivery-more than it does qualityrelated content. Here, we have shown precisely this result: BLAx for the most part eliminated the palatability-specific content in late GC responses without significantly reducing the amount of taste-related content in earlier responses, and without simply reducing the duration of responses to $<1 \mathrm{~s}$ (Fig. $4 B$ ). Infusions of muscimol into CeA or other nearby regions surrounding (but not including) BLA, were much less effective at changing GC responses-CeAx did in fact cause significant number of response changes, an unsurprising result given previous studies demon- strating CeA's influence on brainstem taste responses (Cho et al., 2003; Lundy and Norgren, 2004; Li et al., 2005), but this impact was just one third the magnitude of that observed with BLAx. Thus, the effects described here on firing rates in GC were, in fact, a specific function of the loss of BLA input, and not a function of any incidental inactivation of CeA neurons.

We have previously characterized taste responses in GC as evolving through a sequence of three epochs. In a sequence of studies, we have shown that firing rates progress from reflecting only the presence of stimuli on the tongue in the Early epoch, to taste identity in the Middle epoch, and finally to reflecting palatability of that taste in the Late epoch (Katz et al., 2001; Fontanini and Katz, 2006; Grossman et al., 2008). While the timing of the Middle-to-Late transition can be determined only approximately (most notably, because the gradual transition apparent here is likely an artifact of sudden state-to-state transitions occurring at different times in different trials; Jones et al., 2007; Miller and Katz, 2010), a range of methods brought to bear in these previous studies consistently show it to occur late in the first second of the responses, before the typical onset time of palatability-specific oral behaviors (Travers et al., 1986), and to change with learningrelated changes in taste palatability (Grossman et al., 2008). Lateepoch activity is clearly related to the processing of palatability, and not a simple reflection of palatability-specific behavior.

Our initial hypothesis was that BLAx would only change firing rates in this Late, palatability-processing epoch. Clearly, this hypothesis was simplistic-BLAx affected every aspect of GC taste responses. A look at the density of interconnection makes it clear why this is true: GC neurons make both direct (Saper, 1982; Shi and Cassell, 1998) and indirect (Norgren and Leonard, 1973; Karimnamazi and Travers, 1998) connections with lateral regions of the amygdala. With such a dense, reciprocally connected network, the two regions are likely in a constant state of cooperation; BLAx almost certainly removed a source of tonic, spontaneous activation and inhibition on the cortical neurons.

However, more careful examination of the data revealed that the impact of BLAx did change suddenly around the time of the epochal boundary between identification and palatability coding. While the absolute magnitude of the BLAx impact increased across time in some ensembles and decreased in others, the transition in BLAx-induced differences in firing rate occurred reliably between 0.75 and $1 \mathrm{~s}$ after taste delivery. This timing coincides with the onset of the palatability-processing epoch of GC (Erickson et al., 1994; Katz et al., 2001; Bahar et al., 2004; Jones et al., 2007). Further analysis revealed that the abiding nature of these late changes was indeed a loss of palatability related activity. This finding supports our primary hypothesis that palatability-related responses appear in GC largely because of cooperation with BLA neurons, which produce palatability-related responses one epoch earlier.

Of course, the transmission latency for axons connecting BLA and GC is on the scale of tens of milliseconds at most (Yamamoto et al., 1984; Stone et al., 2011). It is reasonable to ask, therefore, how palatability could possibly be passed from BLA to GC with an epoch-long (hundreds of ms) delay, and why palatabilityrelated firing vanishes from BLA when it appears in GC (Fontanini et al., 2009). While we cannot as of yet offer a definitive answer to these questions, we note that our recent population coding and theoretical modeling studies (Jones et al., 2007; Miller and Katz, 2010) suggest that the taste system may be described as a nonlinear "attractor network," in which it is actually quite reasonable to expect that passage of information from one region to the next occurs only at critical transition points. 
It is worth noting that the taste responses of a subgroup of GC neurons (29\%) were enhanced or "unmasked" by BLAx. The reduction of GC response magnitudes by BLAx may reflect either the removal of the source of taste information to those neurons (i.e., information that reaches GC via BLA rather than via thalamus) or the removal of a modulating influence on taste responses transmitted to GC via the more direct thalamic route; the enhancement of responses, however, almost certainly reflects the latter scenario. That is, while taste information travels from the brainstem to GC via multiple pathways-through the thalamus (Yamamoto, 1984; Kosar et al., 1986), amygdala (Ottersen, 1981), and hypothalamus (Norgren, 1974), for instance-we suspect that the responses we observed to be unmasked by BLAx travel the former route. The fact that these novel responses were "palatability-rich" is consistent with studies showing that decorticated rats can make palatability-related distinctions-that is, that brainstem itself performs basic palatability-related processing (Grill and Norgren, 1978a).

At the whole-dataset level, however, our findings are consistent with studies demonstrating that palatability processing is impaired following BLA lesion (Nachman and Ashe, 1974; Seeley et al., 1993; Murray et al., 1996; Ganaraja and Jeganathan, 2000; Bechara et al., 2003; Lin et al., 2009). In fact, the unmasked responses, which were not suppressed in or removed from any of our analyses, also suggests that we, if anything, under-estimated the amount of palatability-related information that is eliminated by BLAx by including all neurons in all analyses: despite the unmasking of a small set of de novo responses, the overall effect of BLAx was a significant suppression of palatability-related neural firing, emerging at the approximate boundary between the Middle and Late epochs. While BLA is only a part of the distributed system devoted to the processing of palatability, the work presented here reveals that it is nonetheless an important part of that system.

The gustatory system is a complex network whose purpose is to guide the animal's feeding behavior. Our recordings from taste-responsive GC neurons before, during, and after BLAx provide direct evidence that BLA is a critical piece of that network-a necessary component of GC taste processing - and that GC palatability processing in particular is in part a function of BLA activity, as previously suggested. Understanding how signals are passed and interpreted among neural networks is crucial for designing manipulations that allow researchers to determine how spatially separated areas cooperate to process information that subsequently drives behavior. These results of course, investigate only a part of the circuitry involved in the processing of taste perception. Future work will continue to expand our understanding not only of the interplay among the many regions of the gustatory system-and how these interactions function in the context of learning - but other sensory systems as well.

\section{References}

Arikan R, Blake NM, Erinjeri JP, Woolsey TA, Giraud L, Highstein SM (2002) A method to measure the effective spread of focally injected muscimol into the central nervous system with electrophysiology and light microscopy. J Neurosci Methods 118:51-57.

Bahar A, Dudai Y, Ahissar E (2004) Neural signature of taste familiarity in the gustatory cortex of the freely behaving rat. J Neurophysiol 92:3298-3308.

Bauer EP, Paz R, Paré D (2007) Gamma oscillations coordinate amygdalorhinal interactions during learning. J Neurosci 27:9369-9379.

Bechara A, Damasio H, Damasio AR, Lee GP (1999) Different contributions of the human amygdala and ventromedial prefrontal cortex to decisionmaking. J Neurosci 19:5473-5481.
Bechara A, Damasio H, Damasio AR (2003) Role of the amygdala in decision-making. Ann N Y Acad Sci 985:356-369.

Berlau DJ, McGaugh JL (2003) Basolateral amygdala lesions do not prevent memory of context-footshock training. Learn Mem 10:495-502.

Berridge KC (2000) Measuring hedonic impact in animals and infants: microstructure of affective taste reactivity patterns. Neurosci Biobehav Rev 24:173-198.

Breslin PA, Spector AC, Grill HJ (1992) A quantitative comparison of taste reactivity behaviors to sucrose before and after lithium chloride pairings: a unidimensional account of palatability. Behav Neurosci 106:820-836.

Cho YK, Li CS, Smith DV (2003) Descending influences from the lateral hypothalamus and amygdala converge onto medullary taste neurons. Chem Senses 28:155-171.

Di Lorenzo PM, Platt D, Victor JD (2009) Information processing in the parabrachial nucleus of the pons. Ann N Y Acad Sci 1170:365-371.

Erickson RP, Di Lorenzo PM, Woodbury MA (1994) Classification of taste responses in brain stem: membership in fuzzy sets. J Neurophysiol 71:2139-2150.

Escobar ML, Alcocer I, Chao V (1998a) The NMDA receptor antagonist CPP impairs conditioned taste aversion and insular cortex long-term potentiation in vivo. Brain Res 812:246-251.

Escobar ML, Chao V, Bermúdez-Rattoni F (1998b) In vivo long-term potentiation in the insular cortex: NMDA receptor dependence. Brain Res 779:314-319.

Ferreira G, Miranda MI, De la Cruz V, Rodríguez-Ortiz CJ, BermúdezRattoni F (2005) Basolateral amygdala glutamatergic activation enhances taste aversion through NMDA receptor activation in the insular cortex. Eur J Neurosci 22:2596-2604.

Floresco SB, Ghods-Sharifi S, Vexelman C, Magyar O (2006) Dissociable roles for the nucleus accumbens core and shell in regulating set shifting. J Neurosci 26:2449-2457.

Foffani G, Moxon KA (2004) PSTH-based classification of sensory stimuli using ensembles of single neurons. J Neurosci Methods 135:107-120.

Fontanini A, Katz DB (2006) State-dependent modulation of time-varying gustatory responses. J Neurophysiol 96:3183-3193.

Fontanini A, Grossman SE, Figueroa JA, Katz DB (2009) Distinct subtypes of basolateral amygdala taste neurons reflect palatability and reward. J Neurosci 29:2486-2495.

Fortis-Santiago Y, Rodwin BA, Neseliler S, Piette CE, Katz DB (2010) State dependence of olfactory perception as a function of taste cortical inactivation. Nat Neurosci 13:158-159.

Galaverna OG, Seeley RJ, Berridge KC, Grill HJ, Epstein AN, Schulkin J (1993) Lesions of the central nucleus of the amygdala. I: Effects on taste reactivity, taste aversion learning and sodium appetite. Behav Brain Res 59:11-17.

Ganaraja B, Jeganathan PS (2000) Effect of basolateral amygdala and ventromedial hypothalamic lesions on ingestion and taste preference in rat. Indian J Med Res 112:65-70.

Grill HJ, Norgren R (1978a) The taste reactivity test. I. Mimetic responses to gustatory stimuli in neurologically normal rats. Brain Res 143:263-279.

Grill HJ, Norgren R (1978b) The taste reactivity test. II. Mimetic responses to gustatory stimuli in chronic thalamic and chronic decerebrate rats. Brain Res 143:281-297.

Grossman SE, Fontanini A, Wieskopf JS, Katz DB (2008) Learning-related plasticity of temporal coding in simultaneously recorded amygdalacortical ensembles. J Neurosci 28:2864-2873.

Hinkley DV (1971) Inference about the change-point from cumulative sum tests. Biometrika 58:509-523.

Jones LM, Fontanini A, Sadacca BF, Miller P, Katz DB (2007) Natural stimuli evoke dynamic sequences of states in sensory cortical ensembles. Proc Natl Acad Sci U S A 104:18772-18777.

Karimnamazi H, Travers JB (1998) Differential projections from gustatory responsive regions of the parabrachial nucleus to the medulla and forebrain. Brain Res 813:283-302.

Katz DB, Simon SA, Nicolelis MA (2001) Dynamic and multimodal responses of gustatory cortical neurons in awake rats. J Neurosci 21:4478-4489.

Kosar E, Grill HJ, Norgren R (1986) Gustatory cortex in the rat. I. Physiological properties and cytoarchitecture. Brain Res 379:329-341.

Lee IO, Lim ES (2010) Intracisternal or intrathecal glycine, taurine, or muscimol inhibit bicuculline-induced allodynia and thermal hyperalgesia in mice. Acta Pharmacol Sin 31:907-914. 
Li CS, Cho YK, Smith DV (2005) Modulation of parabrachial taste neurons by electrical and chemical stimulation of the lateral hypothalamus and amygdala. J Neurophysiol 93:1183-1196.

Lin JY, Roman C, St Andre J, Reilly S (2009) Taste, olfactory and trigeminal neophobia in rats with forebrain lesions. Brain Res 1251:195-203.

Lundy RF Jr, Norgren R (2004) Activity in the hypothalamus, amygdala, and cortex generates bilateral and convergent modulation of pontine gustatory neurons. J Neurophysiol 91:1143-1157.

Marquis JP, Killcross S, Haddon JE (2007) Inactivation of the prelimbic, but not infralimbic, prefrontal cortex impairs the contextual control of response conflict in rats. Eur J Neurosci 25:559-566.

Martin JH, Ghez C (1999) Pharmacological inactivation in the analysis of the central control of movement. J Neurosci Methods 86:145-159.

Miller P, Katz DB (2010) Stochastic transitions between neural states in taste processing and decision-making. J Neurosci 30:2559-2570.

Moreira CM, Masson S, Carvalho MC, Brandão ML (2007) Exploratory behaviour of rats in the elevated plus-maze is differentially sensitive to inactivation of the basolateral and central amygdaloid nuclei. Brain Res Bull 71:466-474.

Müller M, Fendt M (2006) Temporary inactivation of the medial and basolateral amygdala differentially affects TMT-induced fear behavior in rats. Behav Brain Res 167:57-62.

Murray EA, Gaffan EA, Flint RW Jr (1996) Anterior rhinal cortex and amygdala: dissociation of their contributions to memory and food preference in rhesus monkeys. Behav Neurosci 110:30-42.

Nachman M, Ashe JH (1974) Effects of basolateral amygdala lesions on neophobia, learned taste aversions, and sodium appetite in rats. J Comp Physiol Psychol 87:622-643.

Neseliler S, Narayanan D, Fortis-Santiago Y, Katz DB, Birren SJ (2011) Genetically induced cholinergic hyper-innervation enhances taste learning. Front Syst Neurosci 5:97.

Nicolelis MA, Ghazanfar AA, Faggin BM, Votaw S, Oliveira LM (1997) Reconstructing the engram: simultaneous, multisite, many single neuron recordings. Neuron 18:529-537.

Nieuwenhuis S, Forstmann BU, Wagenmakers EJ (2011) Erroneous analyses of interactions in neuroscience: a problem of significance. Nat Neurosci 14:1105-1107.

Nishijo H, Uwano T, Tamura R, Ono T (1998) Gustatory and multimodal neuronal responses in the amygdala during licking and discrimination of sensory stimuli in awake rats. J Neurophysiol 79:21-36.

Norgren R (1974) Gustatory afferents to ventral forebrain. Brain Res 81:285-295.

Norgren R, Leonard CM (1973) Ascending central gustatory pathways. J Comp Neurol 150:217-237.

Ottersen OP (1981) Afferent connections to the amygdaloid complex of the rat with some observations in the cat. III. Afferents from the lower brain stem. J Comp Neurol 202:335-356.

Ottersen OP (1982) Connections of the amygdala of the rat. IV: Corticoamygdaloid and intraamygdaloid connections as studied with axonal transport of horseradish peroxidase. J Comp Neurol 205:30-48.

Paré D, Collins DR, Pelletier JG (2002) Amygdala oscillations and the consolidation of emotional memories. Trends Cogn Sci 6:306-314.

Parkes SL, Westbrook RF (2010) The basolateral amygdala is critical for the acquisition and extinction of associations between a neutral stimulus and a learned danger signal but not between two neutral stimuli. J Neurosci $30: 12608-12618$.
Paxinos G, Watson C (2007) The rat brain in stereotaxic coordinates, Ed 6. New York: Elsevier/Academic.

Paz R, Bauer EP, Paré D (2009) Measuring correlations and interactions among four simultaneously recorded brain regions during learning. J Neurophysiol 101:2507-2515.

Popa D, Duvarci S, Popescu AT, Léna C, Paré D (2010) Coherent amygdalocortical theta promotes fear memory consolidation during paradoxical sleep. Proc Natl Acad Sci U S A 107:6516-6519.

Rosen AM, Victor JD, Di Lorenzo PM (2011) Temporal coding of taste in the parabrachial nucleus of the pons of the rat. J Neurophysiol 105:1889-1896.

Saddoris MP, Gallagher M, Schoenbaum G (2005) Rapid associative encoding in basolateral amygdala depends on connections with orbitofrontal cortex. Neuron 46:321-331.

Saper CB (1982) Convergence of autonomic and limbic connections in the insular cortex of the rat. J Comp Neurol 210:163-173.

Schoenbaum G, Chiba AA, Gallagher M (1998) Orbitofrontal cortex and basolateral amygdala encode expected outcomes during learning. Nat Neurosci 1:155-159.

Seeley RJ, Galaverna O, Schulkin J, Epstein AN, Grill HJ (1993) Lesions of the central nucleus of the amygdala. II: Effects on intraoral $\mathrm{NaCl}$ intake. Behav Brain Res 59:19-25.

Shi CJ, Cassell MD (1998) Cortical, thalamic, and amygdaloid connections of the anterior and posterior insular cortices. J Comp Neurol 399:440-468.

Spector AC (1995) Gustatory function in the parabrachial nuclei: implications from lesion studies in rats. Rev Neurosci 6:143-175.

Stone ME, Maffei A, Fontanini A (2011) Amygdala stimulation evokes time-varying synaptic responses in the gustatory cortex of anesthetized rats. Front Integr Neurosci 5:3.

Touzani K, Taghzouti K, Velley L (1997) Increase of the aversive value of taste stimuli following ibotenic acid lesion of the central amygdaloid nucleus in the rat. Behav Brain Res 88:133-142.

Travers JB, Travers SP, Norgren R (1987) Gustatory neural processing in the hindbrain. Annu Rev Neurosci 10:595-632.

Travers SP, Pfaffmann C, Norgren R (1986) Convergence of lingual and palatal gustatory neural activity in the nucleus of the solitary tract. Brain Res 365:305-320.

van Duuren E, van der Plasse G, van der Blom R, Joosten RN, Mulder AB, Pennartz CM, Feenstra MG (2007) Pharmacological manipulation of neuronal ensemble activity by reverse microdialysis in freely moving rats: a comparative study of the effects of tetrodotoxin, lidocain, and muscimol. J Pharmacol Exp Ther 323:61-69.

Wang Y, Fontanini A, Katz DB (2006) Temporary basolateral amygdala lesions disrupt acquisition of socially transmitted food preferences in rats. Learn Mem 13:794-800.

Yamamoto T (1984) Taste responses of cortical neurons. Prog Neurobiol 23:273-315.

Yamamoto T, Azuma S, Kawamura Y (1984) Functional relations between the cortical gustatory area and the amygdala: electrophysiological and behavioral studies in rats. Exp Brain Res 56:23-31.

Yamamoto T, Yuyama N, Kato T, Kawamura Y (1985) Gustatory responses of cortical neurons in rats. III. Neural and behavioral measures compared. J Neurophysiol 53:1370-1386. 